The Astrophysical Journal, 673:34-38, 2008 January 20

(C) 2008. The American Astronomical Society. All rights reserved. Printed in U.S.A.

\title{
THE SPATIAL STRUCTURE OF AN ACCRETION DISK
}

\author{
Shawn Poindexter, ${ }^{1}$ Nicholas Morgan, ${ }^{1}$ and Christopher S. KochaneK ${ }^{1}$ \\ Received 2007 June 29; accepted 2007 October 5
}

\begin{abstract}
Based on the microlensing variability of the two-image gravitational lens HE 1104-1805 observed between 0.4 and $8 \mu \mathrm{m}$, we have measured the size and wavelength-dependent structure of the quasar accretion disk. Modeled as a power law in temperature, $T \propto R^{-\beta}$, we measure a $B$-band $(0.13 \mu \mathrm{m}$ in the rest frame $)$ half-light radius of $R_{1 / 2, B}=$ $6.7_{-3.2}^{+6.2} \times 10^{15} \mathrm{~cm}\left(68 \%\right.$ confidence level) and a logarithmic slope of $\beta=0.61_{-0.17}^{+0.21}$ (68\% confidence level) for our standard model with a logarithmic prior on the disk size. Both the scale and the slope are consistent with simple thin disk models where $\beta=3 / 4$ and $R_{1 / 2, B}=5.9 \times 10^{15} \mathrm{~cm}$ for a Shakura-Sunyaev disk radiating at the Eddington limit with $10 \%$ efficiency. The observed fluxes favor a slightly shallower slope, $\beta=0.55_{-0.02}^{+0.03}$, and a significantly smaller size for $\beta=3 / 4$.
\end{abstract}

Subject headings: accretion, accretion disks — gravitational lensing — quasars: individual (HE 1104-1805)

\section{INTRODUCTION}

A simple theoretical prediction for thermally radiating thin accretion disks well outside the inner disk edge is that the temperature diminishes with radius as $T \propto R^{-3 / 4}$ (Shakura \& Sunyaev 1973). This implies a characteristic size at wavelength $\lambda$ of $R_{\lambda} \propto$ $\lambda^{4 / 3}$, where $R_{\lambda}$ is the radius at which $k T=h c / \lambda$. Needless to say, it is unlikely that disks are this simple (e.g., Blaes 2004), but measurement of the size-wavelength scaling would be a fundamental test for any disk theory. While the angular sizes of quasar accretion disks are far too small to be resolved by direct observation, gravitational lenses can serve as natural telescopes to probe accretion disk structure on these scales.

Here we make the first such measurement of this sizewavelength scaling. Our approach, gravitational microlensing of a quasar, will be unfamiliar to the active galactic nucleus (AGN) community, but it is the only method with the necessary spatial resolution available to us for the foreseeable future (see the review by Wambsganss 2006). The stars in the lens galaxy near the image of a multiply imaged quasar generate complex caustic networks with a characteristic scale called the Einstein radius $R_{\mathrm{E}}$ set by the mean stellar mass $\langle M\rangle$. For the lens system we consider here, HE 1104-1805, $\left\langle R_{\mathrm{E}}\right\rangle=3.6 \times 10^{16}\left(\langle M\rangle / h M_{\odot}\right)^{1 / 2} \mathrm{~cm}$. Because the magnification diverges on the caustic curves of the pattern and the source is moving relative to the lens and observer, microlensing allows us to study the spatial structure of anything smaller than $\left\langle R_{\mathrm{E}}\right\rangle$. Using microlensing to probe scales smaller than $R_{\mathrm{E}}$ has been successfully applied to resolve stars in Galactic microlensing events (e.g., Albrow et al. 2001). For quasars, this has been considered analytically or with simulations (e.g., Agol \& Krolik 1999; Goicoechea et al. 2004; Grieger et al. 1991), but the data and algorithms needed to implement the programs have only become available recently (see Kochanek 2004; Kochanek et al 2008).

HE 1104-1805 is a doubly imaged radio-quiet quasar at $z_{S}=$ 2.319 with a separation of $3.15^{\prime \prime}$ (Wisotzki et al. 1993). The lens at $z_{l}=0.729$ was discovered in the near-IR by Courbin et al. (1998) and with HST (Remy et al. 1998; Lehár et al. 2000). Here

\footnotetext{
1 Department of Astronomy and Center for Astro Particle Physics, Ohio State University, 140 West 18th Avenue, Columbus, OH 43210; sdp@astronomy.ohiostate.edu,nmorgan@astronomy.ohio-state.edu, ckochanek@astronomy.ohio-state .edu.
}

we analyze $13 \mathrm{yr}$ of photometric data in 11 bands from the mid-IR to B band using the methods of Kochanek (2004) to measure the wavelength-dependent structure of this quasar modeled as a power law $R_{\lambda} \propto \lambda^{1 / \beta}$. We assume a flat $\Lambda$ CDM cosmological model with $\Omega_{M}=0.3$ and $H_{0}=70 \mathrm{~km} \mathrm{~s}^{-1} \mathrm{Mpc}^{-1}$ and report the disk sizes assuming a mean inclination of $\cos i=1 / 2$. In $\S 2$ we describe the data set and our methods, and $\S 3$ presents our measurement results and our conclusions.

\section{DATA AND METHODS}

We included observations of HE 1104-1805 in 11 bands: $B$, $V, R, I, J, H, K$, and 3.6,4.5, 5.8, and $8.0 \mu \mathrm{m}$. These included our own optical/near-IR data from ANDICAM on the Small and Moderate Aperture Research Telescope System (SMARTS) 1.3 m, Southern Astrophysical Research Telescope (SOAR) near-IR, Hubble Space Telescope (HST), and Spitzer Space Telescope (Spitzer) IRAC data (Poindexter et al. 2007), $R$-band monitoring data by Ofek \& Maoz (2003), the $V$-band monitoring data from Schechter et al. (2003) and Wyrzykowski et al. (2003), and earlier data from Remy et al. (1998), Gil-Merino et al. (2002), Courbin et al. (1998), and Lehár et al. (2000). Where possible, we corrected the light curves for the 152 day time delay between the images we measured in Poindexter et al. (2007). Where we could not, we broadened the photometric uncertainties by 0.07 mag so that the flux ratio uncertainties would be larger by the $0.1 \mathrm{mag}$ shifts we found between time-delay corrected and uncorrected flux ratios. The light curve is plotted along with one of the lightcurve models from our analysis in Figure 1. As pointed out in Poindexter et al. (2007), image A has slowly switched from being bluer than image B to being redder in the optical/near-IR (see Fig. 1), while the mid-IR flux agrees with the flux ratio of the broad emission lines (Wisotzki et al. 1993).

Determining the structure of the disk as a function of wavelength from such data is relatively straightforward. The divergences on the caustics of the microlensing magnification patterns are only renormalized by the finite size of the source quasar because the observed magnification is the convolution of the pattern with the source structure. Thus, larger emission regions will show smaller variability amplitudes because they smooth the patterns more heavily. As we go from the $K$ band to the $B$ band, the radius of a standard thin disk with $R_{\lambda} \propto \lambda^{4 / 3}$ changes by a factor of 8.6 , corresponding to a change in the disk area of almost two orders of magnitude. We see in Figure 1 that the bluer wavelengths 


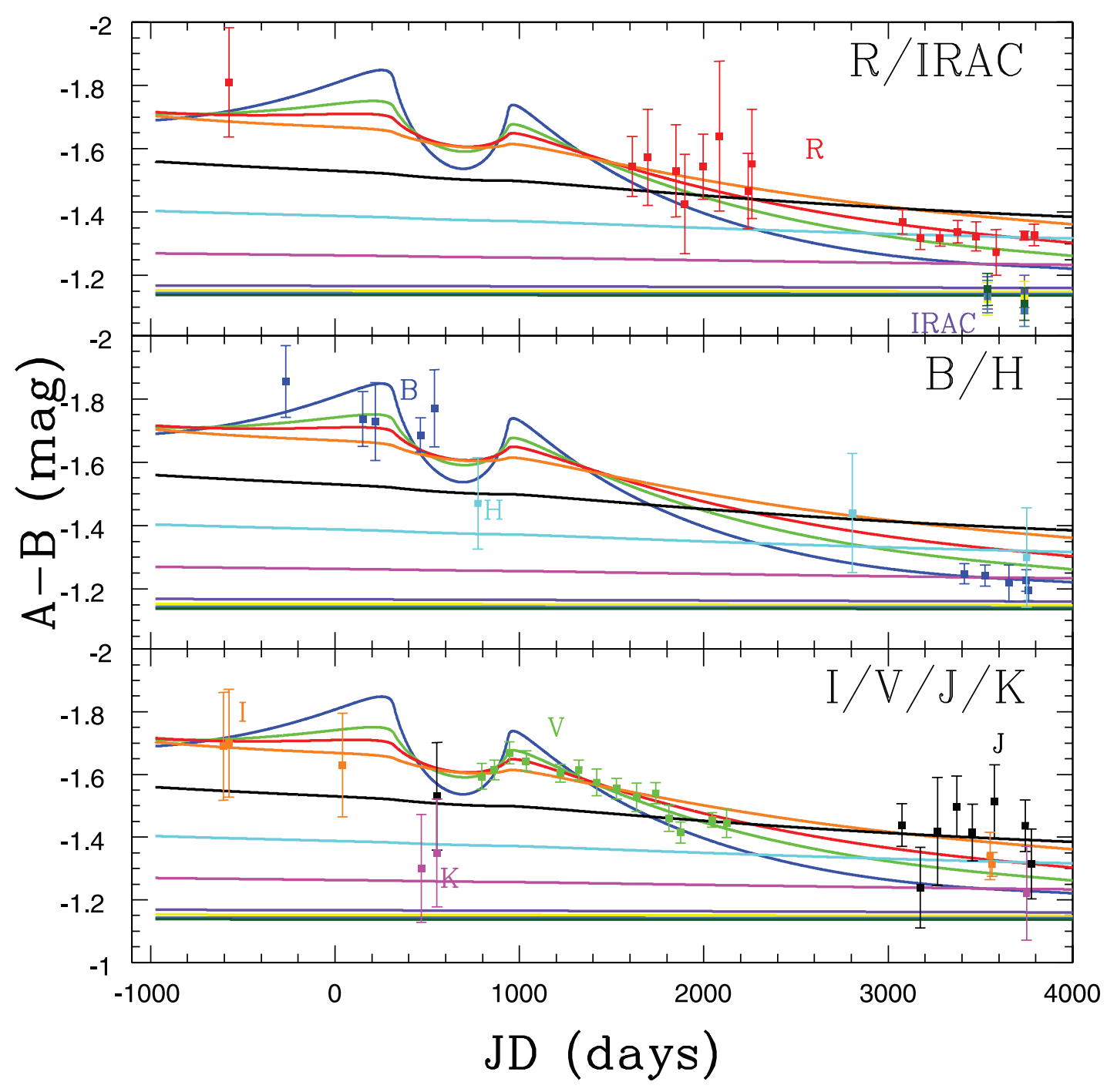

FIG. 1. - The HE 1104-1805 multiband light curves. The curves show a model for the BVRIJHK bands and the four IRAC channels (3.6-8.0 $\mu$ m). For clarity we split the data into three panels and show the $B-, V$-, $R$-, and $J$-band data points in 200 day averages.

show larger variability amplitudes than the red wavelengths, so we immediately know that the blue emission regions are more compact than the red.

Our analysis uses the Bayesian Monte Carlo method of Kochanek (2004) to analyze the data. In essence, we randomly draw large numbers of trial light curves from a range of physical models, fit them to the data, and then use Bayesian statistics to derive probability distributions for the disk structure. We need, however, to discuss the physical variables used in the models over which we average as well as our model for the structure of the accretion disk.

The quantities measured from the microlensing light curve are in "Einstein units" such that the source size and velocity are scaled by $\left(\left\langle M / M_{\odot}\right\rangle\right)^{1 / 2}$. We use a statistical model for the velocities, sometimes including a direct prior on the microlens masses. A velocity prior alone is generally sufficient to give an estimate of the physical source size. In essence if we have $v=\hat{v} \mathrm{~m}^{1 / 2}$ and $r=\hat{r} m^{1 / 2}$, where $m^{1 / 2}=\left\langle M / M_{\odot}\right\rangle$, microlensing constrains $\hat{r}$ and $\hat{v}$ with a rough degeneracy direction that $\hat{r} \propto \hat{v}$. You can get a given level of variability with a small source moving slowly or a big source moving rapidly. The prior on the velocity implies a mass $m^{1 / 2} \propto v / \hat{v}$, so $r=\hat{r} m^{1 / 2} \propto \hat{r} v / \hat{v} \propto v$ and the physical size has little dependence on the mean mass.
We use the lens model sequence from Poindexter et al. (2007), which consists of a de Vaucouleurs model matched to the HST observations embedded in a Navarro-Frenk-White (NFW) halo, to set the shear $\gamma$, convergence $\kappa$, and stellar fraction $\kappa_{*} / \kappa$ for the microlensing magnification patterns. The models were constrained to match the flux ratios of the mid-IR IRAC bands. We used a mass function of $d N / d M \propto M^{-1.3}$ with $M_{\max } / M_{\min }=50$ whose structure is broadly consistent with the Galactic disk mass function of Gould (2000). The lens models were parameterized by $f_{M / L}$, the fraction of a constant mass-to-light ratio $(M / L)$ represented by the visible galaxy. For each of 10 models, $f_{M / L}=$ $0.1,0.2, \ldots, 1.0$, we produce two magnification patterns with an outer dimension of $10 R_{\mathrm{E}}$ and $8192^{2}$ pixels to achieve a pixel scale of $4.4 \times 10^{13}\left(\langle M\rangle / h M_{\odot}\right)^{1 / 2} \mathrm{~cm}^{2}$ pixel $^{-1}$ that is smaller than the gravitational radius $r_{q}=G M / c^{2}=3.5 \times 10^{14} \mathrm{~cm}$ expected for HE 1104-1805 (see $\S 3$ ). We experimented extensively with magnification patterns of different sizes and scales to ensure that our choice of pixel scale and outer dimension did not affect our results. We used the velocity model from Kochanek (2004) where the adopted values are $73 \mathrm{~km} \mathrm{~s}^{-1}$ for our velocity projected onto the lens plane, $308 \mathrm{~km} \mathrm{~s}^{-1}$ for the velocity dispersion of the lens estimated from fitting an isothermal lens model and rms peculiar velocities of 153 and $71 \mathrm{~km} \mathrm{~s}^{-1}$ for the lens and source, respectively. 


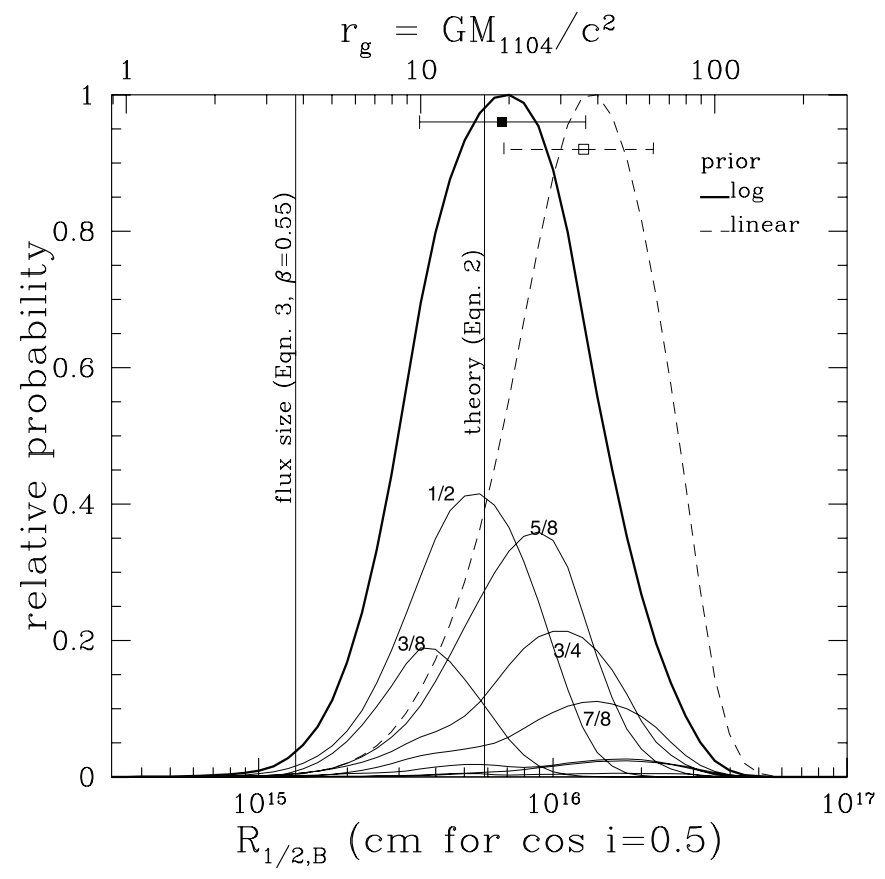

FIG. 2.-Probability distributions for the $B$-band accretion disk half-light radius. The bold solid (dashed) curve is the distribution based on a logarithmic (linear) prior on the disk size. The filled (open) square near the top is the median of the distribution with a logarithmic ( linear) prior along with the $68 \%$ confidence error bars. The lower curves show the contribution from the more significant $\beta$ trials, labeled by their value of $\beta$. The vertical lines show the $B$-band thin disk size predicted from the $I$-band flux (eq. [3]) and the size expected from standard thin disk theory (eq. [2]) for Eddington limited $\left(L / L_{\mathrm{E}}=1\right)$ accretion with $\eta=10 \%$ efficiency.

We can ignore the uncertainties of our velocity and the velocity dispersion of the lens because they are small compared to the rms peculiar velocity of the lens and source even when the velocities are projected into the source plane. We assume a uniform prior on the mean masses of the stars of $0.1 M_{\odot}<\langle M\rangle<1.0 M_{\odot}$, but this has only limited effects on the estimates of the disk size (see Kochanek 2004). Our estimates of the disk properties average over all these parameters.

One limitation of our calculation is the use of fixed magnification patterns. This is a purely practical problem created by the requirement for $\sim 10^{2}$ times more computer memory to use our method with animated magnification patterns in which the stars move. Theoretical studies by Wyithe et al. (2000) indicate that the effect of moving stars can be mimicked by small rescaling of the effective velocity $( \pm 30 \%)$, and such changes would lead to systematic shifts smaller than our present uncertainties.

We modeled the disk as a face-on thermally radiating disk with temperature $T \propto R^{-\beta}$ (e.g., Collier et al. 1998), corresponding to a surface brightness profile of

$$
f_{\nu} \propto \nu^{3}\left[\exp \left(R / R_{\lambda}\right)^{\beta}-1\right]^{-1}
$$

where the size scale is $R_{\lambda}=R_{B}\left(\lambda / \lambda_{B}\right)^{1 / \beta}$ and $R_{B}$ is the disk size at the observed $B$ band (1310 $\AA$ in the rest frame) and $\beta=3 / 4$ for standard thin disk theory. In many cases we report the halflight radius $R_{1 / 2, \lambda}(\beta)=C(\beta) R_{\lambda}(\beta)$, where for $\beta=3 / 4, C=2.44$. While we do not include an inner disk edge of $R_{\text {in }} \simeq 2 r_{g}-6 r_{g}$, the lack of this central hole has little effect on our results unless $\beta \rightarrow 2$ or $R_{B} \simeq R_{\text {in }}$. We tried disk models with profile exponents of $\beta=1 / 4,3 / 8, \ldots, 7 / 4$, making $4 \times 10^{6}$ trial light curves for each value of $\beta$. In our final analysis we use the $3.3 \times 10^{6}$ light

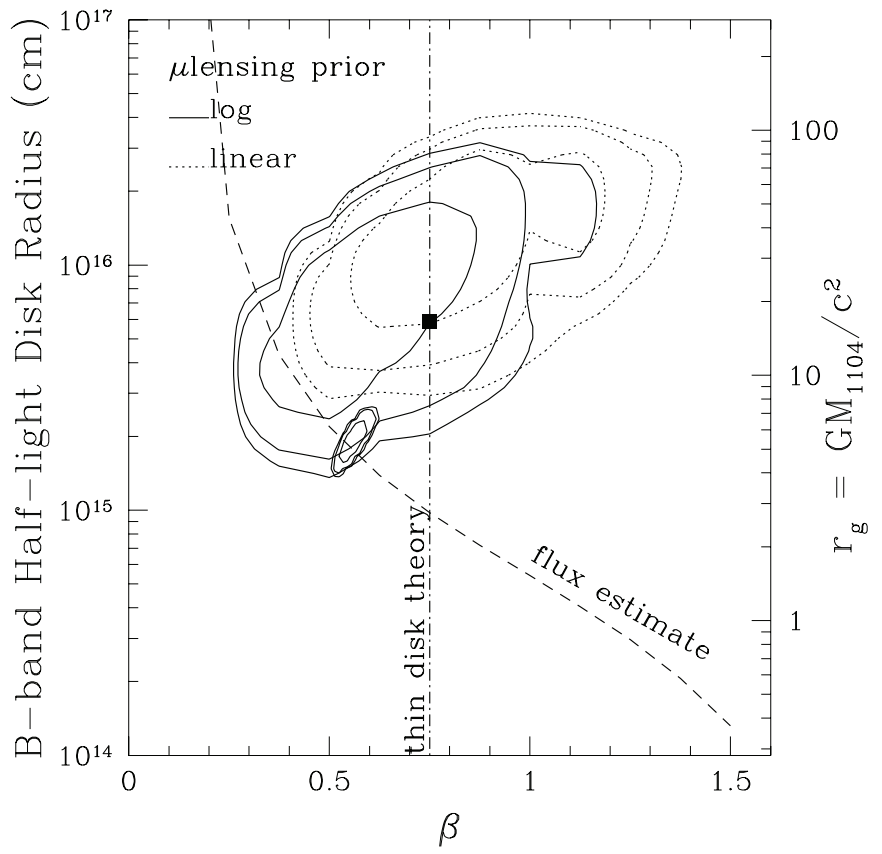

FIG. 3. $-B$-band half-light disk radius $R_{1 / 2, B}$ vs. $\beta$ where $T \propto R^{-\beta}$. The solid (dotted) contours represent the $68 \%, 90 \%$, and $95 \%$ confidence levels of the microlensing measurements assuming a logarithmic (linear) prior on disk size. Small solid contour: $B$-band flux estimate considering a $T \propto R^{-\beta}$ model fit to all the bands. Dashed line: Size estimated from just the $B$-band flux measurement without the constraint on $\beta$ from the other bands. The large solid square at $\beta=3 / 4$ shows the radius predicted by thin disk theory (eq. [2]).

curves that passed a threshold of $\chi^{2} / N_{\text {dof }} \leq 3$ for $N_{\text {dof }}=438$ degrees of freedom. We used both a logarithmic prior, $P\left(R_{\lambda}\right) \propto 1 / R_{\lambda}$, and a linear prior, $P\left(R_{\lambda}\right) \propto$ constant, on the disk size. Generally, a logarithmic prior is preferred for scale-free variables such as $R_{\lambda}$, but for this problem a linear prior may be more appropriate for small source sizes because we are sensitive to the difference between small sizes only during caustic crossings. If, however, we have solutions with the caustic crossing sitting in a gap of the light curve, we will find that $P\left(D \mid R_{\lambda}\right)$ approaches a constant value as $R_{\lambda}$ goes to zero. This leads to a formal divergence in $P\left(R_{\lambda} \mid D\right)$ with a logarithmic prior, suggesting that a linear prior may be more appropriate. In practice, Figures $2-3$ show the results for both options and we use the results for the logarithmic prior in our discussion. For the results we give the value at the median of the probability distribution and the $68 \%(1 \sigma)$ confidence regions.

\section{RESULTS AND DISCUSSION}

We have no difficulty reproducing the observed light curves, including the presently observed color reversal. We illustrate this in Figure 1, where we superpose our best-fitting light curve model on the data.

We find a wavelength-size scaling of the accretion disk of $\beta=0.61_{-0.17}^{+0.21}$ and $\beta=0.89_{-0.16}^{+0.23}$ for the logarithmic and linear priors, respectively (Fig. 4), where $R_{\lambda} \propto \lambda^{1 / \beta}$ and $T \propto R^{-\beta}$. This is consistent with simple thin disk theory $(\beta=3 / 4)$. The $B$-band half-light radius is $R_{1 / 2, B}=6.7_{-3.2}^{+6.2} \times 10^{15} \mathrm{~cm}$ with the logarithmic prior and $R_{1 / 2, B}=1.3_{-0.6}^{+0.9} \times 10^{16} \mathrm{~cm}$ with the linear prior (Figs. 2 and 3). We use the half-light radius rather than $R_{\lambda}$ because it has less covariance with the exponent $\beta$. We can also estimate the sizes for the individual bands, as shown in Figure 4, although these will be highly correlated because the model only has the exponent $\beta$ and one scale length as actual parameters. If we fix $\beta=3 / 4$, then we find that $B$-band size for this case is $R_{1 / 2, B}(\beta=3 / 4)=9.0_{-4.2}^{+6.5} \times 10^{15} \mathrm{~cm}$. 


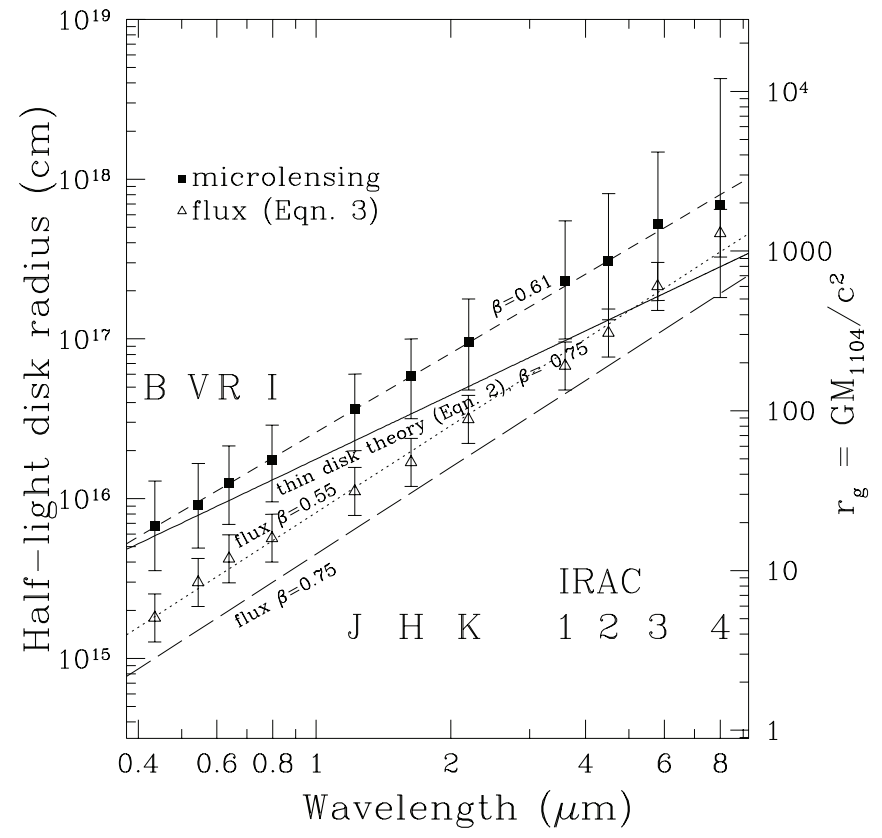

FIG. 4.- Half-light disk radius $R_{1 / 2, \lambda}$ vs. wavelength. The filled squares (open triangles) are the size estimates from microlensing (the source flux, eq. [3]). Note that the microlensing uncertainties are highly correlated because the only two actual variables are $R_{B}$ and $\beta$. The short dashed and dotted lines are the best-fit power laws to these measurements. The long dashed line shows how the normalization of the points would shift if we use $\beta=3 / 4$ rather than the best-fit slope of $\beta=0.55$.

We can compare to thin disk theory only for the case of $\beta=$ $3 / 4$ since there is no simple generalization for alternate values of $\beta$. Based on the $\mathrm{C}$ IV emission line width of HE 1104-1805, Peng et al. (2006) estimated a black hole (BH) mass of $M_{1104}=2.4 \times$ $10^{9} M_{\odot}$, which corresponds to a gravitational radius of $r_{g}=$ $G M / c^{2}=3.5 \times 10^{14} \mathrm{~cm}$. In thin disk theory (Shakura \& Sunyaev 1973), this implies a size scaling of

$$
\begin{aligned}
R_{\lambda} & =\frac{1}{\pi^{2}}\left(\frac{45}{16} \frac{\lambda_{\text {rest }}^{4} r_{g} \dot{M}}{h_{\mathrm{P}}}\right)^{1 / 3} \\
& =\left(1.7 \times 10^{16}\right)\left(\frac{\lambda_{\text {rest }}}{\mu \mathrm{m}}\right)^{4 / 3}\left(\frac{M_{\mathrm{BH}}}{M_{1104}}\right)^{2 / 3}\left(\frac{L}{\eta L_{\mathrm{E}}}\right)^{1 / 3} \mathrm{~cm},
\end{aligned}
$$

where $\eta$ is the radiative efficiency $\left(L=\eta \dot{M} c^{2}\right), h_{\mathrm{P}}$ is the Planck constant, and $L / L_{\mathrm{E}}$ is the fraction of the Eddington luminosity radiated by the quasar. Thus the expected $B$-band (rest frame $0.13 \mu \mathrm{m})$ size for a disk radiating at the Eddington limit $\left(L / L_{\mathrm{E}}\right)$ with $10 \%$ efficiency is $R_{B}=2.4 \times 10^{15} \mathrm{~cm}$ corresponding to a half-light radius of $R_{1 / 2, B}=5.9 \times 10^{15} \mathrm{~cm}$. This agrees well with our measurement (Figs. 2, 3, and 4). For the $\beta=3 / 4$ model the disk scale length is significantly larger than the gravitational radius $\left(R_{1 / 2, B} \sim 20 r_{g}\right)$ and corrections for the inner edge of the disk will be modest. Particularly if we allow for uncertainties in the black hole mass estimate $(\sim 0.3 \mathrm{dex})$, there is good agreement with the simplest possible thin disk model.

The magnification-corrected flux of the quasar provides a second comparison scale under the assumption that the disk is thermally radiating. If the quasar has a magnification corrected magnitude of $m$, then

$$
\begin{aligned}
R_{\lambda}(\beta) \simeq & \frac{2.8 \times 10^{15}}{h \sqrt{K(\beta) \cos i}} \frac{D_{\mathrm{OS}}}{r_{H}}\left(\frac{\lambda_{\mathrm{obs}}}{\mu \mathrm{m}}\right)^{3 / 2} \\
& \times\left(\frac{\mathrm{zpt}}{2409 \mathrm{Jy}}\right)^{1 / 2} 10^{-0.2(m-19)} \mathrm{cm} .
\end{aligned}
$$

where $\lambda_{\text {obs }}$ is the observed wavelength, zpt is the filter zero point (normalized to the $I$ band), and

$$
K(\beta)=\frac{1}{2.58} \int_{0}^{\infty} u d u\left[\exp \left(u^{\beta}\right)-1\right]^{-1}
$$

is the $\beta$-dependent term due to the temperature profile normalized so that $K(\beta=3 / 4)=1$. Assuming the magnifications of images $A$ and $B$ are 11.5 and 4 , respectively (as found in the bestfit macro model in Poindexter et al. 2007), we calculated the magnification-corrected source magnitude in each of the 11 bands to estimate the disk size versus wavelength (Fig. 4). For the $H, I$, and $V$ bands, we used the HST observations of Lehár et al. (2000). The mid-IR magnitudes are from Spitzer (Poindexter et al. 2007). We calibrated the SMARTS $B / R$ and $J / K$ data using the Guide Star Catalog and 2MASS, respectively.

The sizes estimated from the flux are very well fit by a power law (see Fig. 4) with a slope equivalent to $\beta=0.55_{-0.02}^{+0.03}$ when we assume a magnification uncertainty of a factor of 2 . While this slope is consistent with our microlensing results, the size scale of $R_{1 / 2, B}=1.8_{-0.5}^{+0.7} \times 10^{15}$ is smaller by a factor of 4 than our standard estimate. This discrepancy depends on the value of $\beta$, with a flatter temperature profile showing less of a difference. While there is little extinction in the lens, 2.9 mag of $B$-band $(0.31 \mu \mathrm{m}$ rest frame) extinction in the source would reconcile the flux and microlensing size estimates. However, with such an extinction, neither Small Magellanic Cloud (Gordon et al. 2003) nor AGN (Gaskell et al. 2004) extinction curves can reconcile the two estimates at all wavelengths. Nonetheless absorption in the source could be a partial explanation.

The general relationship we find between the microlensing, thin disk, and flux sizes seems to be typical. Pooley et al. (2007) noted qualitatively that microlensing sizes tended to be larger than expected from the optical flux and thin disk models. Morgan et al. (2007) showed quantitatively that the microlensing sizes scaled as expected with $\mathrm{BH}$ mass $\left(R_{\lambda} \propto M^{2 / 3}\right)$ and were consistent with being proportional to the flux sizes, but that the absolute scales of the microlensing sizes were slightly larger than the thin disk sizes and considerably larger than the flux sizes. Our results here suggest that part of the solution may be that the effective temperature profile is somewhat shallower than $T \propto R^{-3 / 4}$.

Several local estimates (Collier et al. 1999; Sergeev et al. 2005) have found UV-optical wavelength-dependent time delays of nearby AGNs consistent with $T \propto R^{-3 / 4}$. They also found the flux discrepancy, but phrased the problem as needing to put the systems at higher than expected distances (through a low value of $H_{0}$ ) in order to reconcile the model disk surface brightness with the observed flux.

Our current disk model is a face-on, thermally radiating disk without the central temperature depression created by the inner edge of the accretion disk (eq. [1]). Omitting the inner edge has little effect because at fixed wavelength little flux is radiated there and the finite resolution of the magnification patterns eliminates the formal surface brightness divergence in equation (1). It is expected from theoretical work that microlensing is primarily sensitive to an effective smoothing area, and the resulting size estimate is only weekly sensitive to the true surface brightness profile (e.g., Mortonson et al. 2005; Congdon et al. 2007). Nonetheless, a clear next step is to begin interpreting the microlensing results using more realistic thin disk models (e.g., Hubeny et al. 2001 ) to see whether adding such details alters the basic picture. The present results suggest that part of the solution may be to alter the temperature profile of the disk, perhaps through irradiation of the outer regions by the inner regions. 
We thank R. Pogge, E. Agol, C. Morgan, and X. Dai for discussions on microlensing and accretion disks, and suggestions for improving the manuscript. Support for this work was provided by NASA through an award GRT00003172 issued by JPL/ Caltech and NSF grant AST-0708082. This research is based on observations obtained with the Small and Moderate Aperture Research Telescope System (SMARTS). This work is based in part on observations made with the Spitzer Space Telescope, which is operated by the Jet Propulsion Laboratory, California Institute of Technology under a contract with NASA. Based on observations made with the NASA/ESA Hubble Space Telescope, obtained at the Space Telescope Institute. STScI is operated by the association of Universities for Research in Astronomy, Inc., under the NASA contract NAS5-26555. This publication makes use of data products from the Two Micron All Sky Survey, which is a joint project of the University of Massachusetts and the Infrared Processing and Analysis Center/California Institute of Technology, funded by NASA and NSF. The Guide Star Catalogue-II is a joint project of the STScI and the Osservatorio Astronomico di Torino. STScI is operated by the AURA, for NASA under contract NAS5-26555. The participation of the Osservatorio Astronomico di Torino is supported by the Italian Council for Research in Astronomy. Additional support is provided by ESO, Space Telescope European Coordinating Facility, the International GEMINI project, and the ESA Astrophysics Division.
Agol, E., \& Krolik, J. 1999, ApJ, 524, 49

Albrow, M., et al. 2001, ApJ, 550, L173

Blaes, O. M. 2004, in Accretion Discs, Jets and High Energy Phenomena in Astrophysics, ed. V. Beskin et al. (Berlin: Springer), 137

Collier, S., Horne, K., Wanders, I., \& Peterson, B. M. 1999, MNRAS, 302, L24

Collier, S. J., et al. 1998, ApJ, 500, 162

Congdon, A. B., Keeton, C. R., \& Osmer, S. J. 2007, MNRAS, 376, 263

Courbin, F., Lidman, C., \& Magain, P. 1998, A\&A, 330, 57

Gaskell, C. M., Goosmann, R. W., Antonucci, R. R. J., \& Whysong, D. H. 2004, ApJ, 616, 147

Gil-Merino, R., Wisotzki, L., \& Wambsganss, J. 2002, A\&A, 381, 428

Goicoechea, L. J., Shalyapin, V., González-Cadelo, J., \& Oscoz, A. 2004, A\&A, 425, 475

Gordon, K. D., Clayton, G. C., Misselt, K. A., Landolt, A. U., \& Wolff, M. J. 2003, ApJ, 594, 279

Gould, A. 2000, ApJ, 535, 928

Grieger, B., Kayser, R., \& Schramm, T. 1991, A\&A, 252, 508

Hubeny, I., Blaes, O., Krolik, J. H., \& Agol, E. 2001, ApJ, 559, 680

Kochanek, C. S. 2004, ApJ, 605, 58

Kochanek, C. S., Dai, X., Morgan, C., Morgan, N., Poindexter, S., \& Chartas, G. 2008, in Statistical Challenges in Modern Astronomy IV, ed. G. J. Babu \& E. D. Feigelson (San Francisco: ASP), in press (astro-ph/0609112)

\section{REFERENCES}

Lehár, J., et al. 2000, ApJ, 536, 584

Morgan, C., Kochanek, C. S., Morgan, N. D., \& Falco, E. E. 2007, ApJL, submitted (arXiv:0707.0305)

Mortonson, M. J., Schechter, P. L., \& Wambsganss, J. 2005, ApJ, 628, 594

Ofek, E. O., \& Maoz, D. 2003, ApJ, 594, 101

Peng, C. Y., Impey, C. D., Rix, H.-W., Kochanek, C. S., Keeton, C. R., Falco, E. E., Lehár, J., \& McLeod, B. A. 2006, ApJ, 649, 616

Poindexter, S., Morgan, N., Kochanek, C. S., \& Falco, E. E. 2007, ApJ, 660, 146 Pooley, D., Blackburne, J. A., Rappaport, S., \& Schechter, P. L. 2007, ApJ, 661, 19

Remy, M., Claeskens, J.-F., Surdej, J., Hjorth, J., Refsdal, S., Wucknitz, O., Sørensen, A. N., \& Grundahl, F. 1998, NewA, 3, 379

Schechter, P. L., et al. 2003, ApJ, 584, 657

Sergeev, S. G., Doroshenko, V. T., Golubinskiy, Y. V., Merkulova, N. I., \& Sergeeva, E. A. 2005, ApJ, 622, 129

Shakura, N. I., \& Sunyaev, R. A. 1973, A\&A, 24, 337

Wambsganss, J. 2006, in Proc. Saas-Fee Advanced Course 33, Gravitational Lensing: Strong Weak and Micro, ed. G. Meylan, P. North, \& P. Jetzer (Berlin: Springer), 453

Wisotzki, L., Köhler, R., Kayser, R., \& Reimers, D. 1993, A\&A, 278, L15 Wyithe, J. S. B., Webster, R. L., \& Turner, E. L. 2000, MNRAS, 312, 843 Wyrzykowski, Ł. et al. 2003, Acta Astron., 53, 229 